\title{
El derecho en América Latina: Un mapa para el pensamiento jurídico del siglo XXI
}

\author{
Autor: César Rodríguez Garavito (coord.), \\ Editorial Siglo XXI, Buenos Aires, 2011.
}

Por: Cuitlahuac CASTILLO CAMARENA ${ }^{1}$

El Derecho en Latinoamérica ha sido un punto de referencia teórico novedoso en el que se ha articulado una tradición europea de pensarlo heredada de nuestras historias como colonias, eso es evidente. La influencia imperante de Kelsen en nuestro ordenamiento jurídico, en la educación y promoción del derecho, así como la importación de diversas teorías de pensadores europeos ajenos a nuestro contexto, han sido uno de los diversos talones de Aquiles en el desarrollo de la ciencia jurídica regional.

Entender que el derecho es el producto de esas influencias estructurales podría situarnos ventajosamente frente a un sin número de problemáticas que en el área jurídica se presentan. ¿Qué ha ofrecido el derecho como ciencia en los últimos años desde nuestra región? ¿Se ha salido de los cánones tradicionales de teorías que poco coincidían con las necesidades de nuestro contexto? ¿Qué ha innovado? El punto central es reconocer el bagaje teórico que se ha generado en la zona ¿Qué ha surgido? ¿De qué fenómenos da cuenta?

Nuestro contexto en este momento está pasando por una interesante etapa de transformaciones: un robustecimiento de derechos humanos en las constituciones de la mayoría de la países en Latinoamérica, una singular alternancia de regímenes dictatoriales a democracias débiles, un desarrollo rico en derecho internacional y un ordenamiento jurídico supranacional que ya es parte de nuestro bagaje jurídico de libertades como el caso del Sistema Interamericano. También se ha presentado una concepción distinta del Poder Judicial y de los Tribunales Constitucionales de las naciones como poderes novedosamente autónomos e independientes, en compañía de diversos conflictos más totalizadores como la violencia

\footnotetext{
1 Alumno de la Licenciatura en Derecho por la Universidad de Guanajuato; Miembro del Programa Universitario de Investigaciones Jurídicas de la División de Derecho, Política y Gobierno de la Universidad de Guanajuato.

${ }^{2}$ Respecto a este punto se ha criticado en pocas ocasiones la forma en la que se enseña el derecho en América Latina. Algunos modelos de educación jurídica han versado sobre distintas maneras de transmitir el conocimiento jurídico teniendo ello una repercusión en la consolidación de un Estado de Derecho. Por ejemplo se me viene a la mente la forma estadounidense de enseñar derecho: por medio de casos específicos y prácticos y un método mayéutico de aprendizaje. También algunos autores como los pertenecientes a los estudios críticos del derecho han llegado a ver la enseñanza del derecho como un acto político al determinar qué tipo de abogados se están creando y qué tipo de prácticas jurídicas se van a perpetuar. Para ahondar en este punto un autor imperdible sin duda es: MADRAZO, Alejandro, “¿Qué?, ¿Cómo? y ¿Para qué?: Análisis y crítica al modelo tradicional de enseñanza del Derecho en México". Revista sobre enseñanza del Derecho, Año 4, No. 7, 2006.
} 
criminal desatada por la producción de drogas ilegales, la debilidad de las instituciones representativas y la carente legitimidad de figuras presidencialistas predominantes en los países del sur americano, son algunos de los temas que deben de pensarse por los operadores, creadores, académicos y críticos del derecho.

Esta coordinación de César Rodríguez Garavito reúne trabajos de juristas y académicos que han realizado distintas investigaciones y proyectos sobre el derecho y la articulación teórica y práctica que ha tenido en algunos países pertenecientes a Latinoamérica.

Lo imprescindible de la obra es justamente eso, El Derecho en América Latina da cuenta de un mapeo que ya era necesario: la teoría jurídica en Latinoamérica existe, está vigente y sin duda está intentando develar, explicar y fomentar rupturas en los paradigmas de derecho clásico que ya se habían gestado en la teoría y práctica de la región, y que poco explicaban lo que sucedía fuera de la academia.

La obra tiene cuatro partes, la primera: El contexto: desigualdad, violencia y globalización, la segunda: Constitución, estado de derecho y cultura jurídica, la tercera: Derechos Humanos, cortes y derecho internacional y finalmente la cuarta: Los retos de la justicia. Desde la estructura del libro se observa que las partes del libro mapean aspectos elementales para evaluar el pensamiento jurídico de la región.

En el primer apartado El contexto: desigualdad, violencia y globalización la coordinación nos presenta tres ensayos que develan la realidad en Latinoamérica:

- En "Desigualdad estructural y Estado de derecho" Oscar Vilhena Vieira expone las razones por las cuáles las personas respetan las normas: razones instrumentales, cognitivas y morales. Las instrumentales se refieren a aquellas recompensas y castigos por respetar o no la ley, las cognitivas a la comprensión -de las personas que están sujetas a respetar la ley deberían tener- sobre conceptos como derechos, obligaciones y normas. Finalmente las terceras se refieren al esquema de valores que fue creado contractualmente por los seres humanos que integran una sociedad. Vilhena expone estos tres niveles de razonamientos para respetar normas siendo pertinente un análisis de cuáles están fallando en una zona azotada por la violencia, el incumplimiento y la corrupción.

- El segundo capítulo ¿Constitución o barbarie? Cómo repensar el derecho en las zonas "sin ley" Julieta Lemaitre trae a la discusión algo imperdible: ¿Cuál es la relación entre el derecho que enseñamos en las aulas universitarias y la violencia que sucede fuera del salón de clase? ¿Cómo es que la filosofía y la teoría del derecho puede responder a esas zonas precarias? Hace una dicotomía entre civilización y barbarie. Lo cierto es que esos estados vacíos de teoría no van más allá de la reflexión de calificar esos lugares violentos como estados sin ley, se habla más de la usencia de legalidad que de la propia violencia que allí florece. ¿Qué sucede en las montañas colombianas y en las favelas de Brasil? Lemaitre trae de nueva cuenta la discusión en las teorías liberales del derecho y las marxistas o estudios críticos, ambos planos teóricos no pueden pensar qué sucede con el derecho en zonas violentas más allá de afirmar que es débil. ¿Cómo repensar el derecho? Ofrece algunos argumentos desde el postestructuralismo de la teoría social cuya concepción del derecho no se limita a la dicotomía de limitar violencia y de instrumento de opresión presentada por teorías marxistas críticas del derecho, sino que ve al derecho como un fenómeno sujeto a su propia historia, sus propias transformaciones, prácticas y significados sociales. 
- El tercer ensayo Navegando la globalización: un mapamundi para el estudio y la práctica del derecho en América Latina, César Rodríguez Garavito despliega un mapa para entender la cartografía y globalización jurídica. Distingue entre una práctica jurídica regional de tintes postwestfalianos mientras que el pensamiento jurídico está centrado en el dilema Estado-nación de carácter totalmente westfaliano. Existe un problema de encuadre producto de la práctica y de la teoría jurídica en la región. Rodríguez Garavito se centra en un sector específico del campo jurídico en la región: el neoconstitucionalismo latinoamericano, es decir, todos los abogados, académicos que están centrándose en una concepción garantista de los derechos humanos, del Estado de Derecho, de la justicia social y el rol activo de los jueces para concretarla.

- Lo pertinente de enfocarse en el neoconstitucionalismo es su carácter de pensamiento globalizado, todo este bagaje teórico ha generado un conocimiento que ha llegado muy lejos y ha sido analizado críticamente en diversos puntos de Latinoamérica. La relevancia de un enfoque así que incluya el ingrediente "globalización" con el conocimiento jurídico puede aportarnos una lente bifocal que da cuenta del proceso epistemológico compartido en distintos de países, de su expansión y de su estudio desde diversos puntos geográficos.

En la segunda parte del libro encontramos once ensayos:

- En Pensando la reforma constitucional en América Latina Roberto Gargarella plantea algunas discusiones sobre las estructuras constitucionales vigentes en esta parte del continente. Afirma que nos movemos en un marco elitista institucional que fue plasmado en las constituciones instruyendo un poder evidentemente contramayoritario. Gargarella menciona que en algunas constituciones la izquierda intentó incorporar derechos de corte progresistas -es el caso de México- en la Constitución de 1917. Señala que "[e]s cierto que en un principio, y por algunas décadas, muchas de esas cláusulas quedaron como "derechos dormidos" aparentemente inoperantes, meramente consagrados en el "papel" de la Constitución. Sin embargo, también es cierto que en muchos casos, y con el paso del tiempo, esos derechos demostraron tener capacidad para "despertarse" y "activarse" acompañando la sucesión de cambios en la correlación de fuerzas políticas imperantes" (p.96). Gargarella también apela por plantear una modificación a las constituciones que incluyan lo que Rawls denominó como "la estructura básica" de la sociedad, o de lo contrario esos derechos seguirán durmiendo. Admite que parte de lo que se alimenta el derecho corresponde a otras áreas del conocimiento como la economía y la política y que estas fueron fundamentales en las reformas constitucionales y por consecuencia también en su aplicación.

- Rodrigo Uprimny en Las transformaciones constitucionales recientes en América Latina: tendencias y desafíos en un ejercicio de derecho comparado señala las diferencias y similitudes de las constituciones teniendo como base estructural tres razones: en primer lugar el origen y la naturaleza del proceso constituyente dado que algunas constituciones fueron el resultado de la caída de dictaduras militares y otras buscaron continuar con regímenes democráticos. En segundo lugar la intensidad de las reformas constitucionales y qué cambios introdujeron al ordenamiento jurídico y político y con qué trascendencia impactaron a los mismos. Y tercero, la orientación que tienen esas reformas, si son de corte neoliberal o de constitucionalismo transformador con orientación anticapitalista. 
- El sexto ensayo El horizonte del constitucionalismo pluralista: del multiculturalismo a la descolonización Raquel Z. Yrigoyen Fajardo devela tres ciclos de reformas constitucionales, de 1980 a 2010, sobre derechos de reconocimiento a la diversidad cultural y de pueblos indígenas. Estos cambios impactan de forma central al núcleo de definición del estado que los primeros movimientos de constitucionalismo asentaron en proyectos políticos "neocoloniales de sujeción indígena" (p.139). Expone cómo han acogido los países estos conceptos dentro de sus marcos normativos, qué diferencias han tenido en países como Bolivia y Ecuador donde la población es mayoritariamente indígena de países que no son predominantemente indígenas. Expone los límites y problemas de los derechos en cada país, los cambios emergentes respecto a la relación del ser humano y la naturaleza (Pacha Mama en la Constitución de Ecuador).

- Mauricio García Villegas escribe Ineficacia del derecho y cultura del incumplimiento de reglas en América Latina, describe tres tipos de subjetividades o mentalidades incumplidoras, es decir tres tipos de personajes distintos que pueden constituir arquetipos para entender las razones y contextos en los cuáles actúan incumpliendo la ley. En primer lugar habla de "el vivo" que es el tipo de incumplidor más común en América Latina, si se aprovecha de los demás y de las normas es por conveniencia, con el fin de satisfacer su propio beneficio personal. Los costos de incumplir las leyes son más bajos comparados con los resultados obtenidos. En segundo lugar identifica al "rebelde" quien niega la legitimidad de la autoridad que crea o impone la norma y por ello se siente con el derecho de no cumplirla. El tercero que denomina como "arrogante" dado que si acepta la norma pero él, cree que debido a su posición y su persona tiene derecho a no cumpirla, el arrogante tiene cierto espíritu elitista. El cuarto lo denomina como taimado que contiene combina la estrategia del vivo y del rebelde. Finalmente el déspota que considera valores extralegales combinando la arrogancia, el abuso del poder y la viveza. En este ensayo García Villegas describe estos personajes con el fin de identificar los sujetos que en nuestra cultura es predominantemente el transgresor de la norma y explicar por qué nuestro contexto cultural lo ha determinado así.

- El octavo ensayo titulado Lusus naturae, Martin Bohmer señala algo crucial para le teoría jurídica: "Volver a leer a los clásicos de la filosofía del derecho en Latinoamérica todavía equivale a leer tres pensadores extranjeros a la región: un austríaco expatriado, un inglés y un estadounidense" refiriéndose a Hans Kelsen, H.L.A. Hart y Ronald Dworkin. El objetivo de Bohmer es sencillo, pero profundo: explicar qué teoría del derecho escribieron ellos, bajo qué situación y contexto se encontraban esos autores y cuáles eran los problemas que buscaban solucionar. Esto en la medida de poner sin tregua sobre la mesa las discusiones teóricas que los utilizan en América Latina para la resolución de problemas locales. Desglosa y hace una crítica respecto a los modelos educativos en Latinoamérica, las problemáticas locales y las opciones teóricas construidas para resolverlos. Los comentarios a estos tres autores sin duda son relevantes por la influencia que tienen en la región.

La segunda parte dedicada a Derechos Humanos, cortes y derecho internacional contiene los ensayos:

- Autonomía y subsidiaridad. El Sistema Interamericano de Derechos Humanos frente a los sistemas de justicia nacionales. Abramovich hace una revisión a la influencia que ha 
tenido la construcción del Sistema Interamericano de Derechos Humanos (SIDH) en los estados que pactaron la sujeción a la Convención Interamericana. Así mismo de cómo los estados han acoplado el ordenamiento internacional internamente y también qué se ha criticado respecto al tipo de intervención realizada por el SIDH.

- En Justicia constitucional y protección de derechos en América Latina: el debate sobre la regionalización del activismo, Francisca Pou Giménez en donde explica cómo las declaraciones constitucionales de la región así como las internacionales han encaminado la protección judicial de los derechos, incorporando derechos de primera, segunda y tercera generación así como instrumentos para su interpretación. Todo ello ha venido dando una exigencia distinta respecto a lo que los jueces pueden hacer, en palabras de Pou "implicarse responsablemente en la dinámica regionalizadora exige, paradójicamente, desregionalizar parte del análisis y hacerlo sensible a factores institucionales, políticos, personales, culturales e ideológicos cuyas dinámicas de interacción a nivel micro es importante conocer para que la judicialización de los derechos sea verdaderamente emancipadora y no conduzca a la arbitrariedad de los jueces, a los litigios elitistas o a cualquier otra de las dolencias características de la "mala” judicialización” (p. 245).

- Jorge Contesse Singh en Constitucionalismo interamericano: algunas notas sobre las dinámicas de creación e internalización de los derechos humanos devela algunas de las tensiones y dinámicas existentes entre el ordenamiento interamericano y el interno. El análisis que propone busca determina los espacios de trabajo y de intervención que permiten robustecer los derechos vía al interior o exterior de los países.

- Catalina Botero Marino en Problemas persistentes y desafíos emergentes en materia de libertad de expresión en las Américas trae a cuenta discusiones regionales respecto al derecho de libertad de expresión, consciente del renacer democrático en las Américas. Despliega y explica una serie de desafíos en la materia: (a) la creciente violencia contra periodistas, (b) el uso del derecho penal para criminalizar la expresión disidente o crítica, (c) la resistente cultura del secreto, no existiendo mecanismos efectos de libre acceso a la información, (d) los mecanismos múltiples y diversos de censura previa y (e) la ausencia de pluralismos y diversidad en el debate público y en los procesos comunicativos.

- La relevancia de pensar y repensar el derecho a la libre expresión es imprescindible dado las recientes muertes brutales de periodistas en la región latinoamericana, no requiere bastante argumentación para convencerse de la importancia crucial de este derecho para nuestros proyectos políticos democráticos.

- Rachel Sieder en el ensayo número trece sobre Pueblos indígenas y derecho (s) en América Latina señala algunos de los procesos de judicialización que actualmente enfrentan los pueblos indígenas para la efectividad de sus derechos. Da cuenta de que en la mayoría de los países los jueces no están comprometidos plenamente con los derechos colectivos y que incluso llegan a cuestionarlos. En otros países justamente las discusiones respecto a derechos colectivos de los prueblos indígenas han versado sobre el desarrollo del modelo económico dominante, y sus impulsores no han sido los poderes judiciales sino legislativos y ejecutivos. Sieder señala: "Aun cuando se gane un caso de litigio estratégico, no 
necesariamente se garantizará que las políticas públicas o acciones de los actores privados realmente cambien de forma sustantiva."

- En el ensayo número catorce Ronaldo Porto Macedo escribe Derecho social, medio ambiente y desarrollo. Reflexiones en torno a un caso exitoso en el que señala algo imperdible en las recientes discusiones sobre derechos, efectividad y políticas públicas: la articulación que la demanda del derecho ha tenido sobre las desiciones de los jueces, y cómo es que estas han dado como resultado políticas públicas. Este punto ha sido bastante debatido, y lo ejemplifica perfectamente con un caso paradigmático acontecido en Toritama, Brasil. El asunto es simple: Toritama es una ciudad en donde existía una industria de cuero, posteriormente incluyo empresas de confección y lavanderías. En casi todas las operaciones que realizaba esta industria existía contaminación de agua, asociaciones civiles tuvieron conocimiento de ello y comenzaron a entablar demandas que terminaron dando como resultado políticas públicas ambientalistas que fueron gradualmente incluidas en los derechos y obligaciones de las empresas en esa región.

La cuarta parte de esta obra Los retos de la Justicia incluyen tres ensayos:

- Leonardo Avritzer y Fernando Filgueiras escriben Control y observación Judicial: la publicitación de las acciones y trayectorias de los jueces del Supremo Tribunal Federal en donde revisan algunos aspectos cruciales de cómo se venía entendiendo el Poder Judicial en Brasil. Están de acuerdo en que se requiere un proyecto académico de observación de justicia que tendrá que entender y evaluar el nuevo papel que tienen los poderes judiciales, específicamente qué tipo de actores han tenido acceso a él (asociaciones civiles) y qué derechos han tenido como resultado, también una evaluación sobre la relación que entabla con el Poder Legislativo, ya que la nueva relación que tienen implica un debilitamiento de este último por parte del primero. Y finalmente la importancia de ver qué forma equilibrada puede tener el Poder Judicial respecto a los demás poderes.

- En Inseguridad ciudadana y derechos humanos: por la deconstrucción de un discurso securitista y hacia un nuevo derecho penal Ramiro Ávila Santamaría discute con el derecho penal y su concepción actual bajo el prisma de los derechos humanos, y sobre la posible transformación del discurso penalista bajo este esquema. Discute y deconstruye algunos discursos legitimadores de los derechos humanos, y apela por la implementación de un proyecto penal de corte constitucional que pone como centro y fin los derechos humanos.

-El último ensayo de Luciana Gross Cunha, Medir la justicia: el caso del índice de confianza en la justicia (ICJ) en Brasil muestra un estudio empírico sobre la opinión que tiene la población brasileña sobre el Poder Judicial, la efectividad respecto algunos derechos como los laborales, de vecindad, de consumidor y de prestación de derechos. Entre los diversos datos que posee este ensayo, uno respecto a los gastos de la justicia señala que pasaron de 64,23 reales por habitante en 2004 a 100,56 reales por habitante en 2008. Estudios como estos son cruciales si como estados democráticos y constitucionales pretendemos someter a escrutinio, transparencia y Accountability a las instituciones de todos los poderes.

La importancia vigente de este texto es la seriedad y la amplitud con la que mapea el pensamiento jurídico de la región. Los autores son profesores excelsos en cada una de sus áreas 
y sin duda alguna son un inicio para ahondar en los diversos puntos de interés que convergen la problemática común en Latinoamérica: desigualdad estructural, débiles democracias y estados de derecho, activismo judicial, revoluciones de derechos, efectividad de los derechos, SIDH y ordenamientos constitucionales internos, pluralismo y multiculturalismo, entre otros puntos clave para entender la compleja transición que nuestros ordenamientos están pasando. 